\title{
Sistema intrauterino em localização extrauterina
}

\author{
Intrauterine system in extrauterine location \\ Sistema intrauterino en localización extrauterina
}

Recebido: 02/04/2021 | Revisado: 11/04/2021 | Aceito: 20/04/2021 | Publicado: 06/05/2021

João Paulo Pereira Leão
ORCID: https://orcid.org/0000-0001-7630-5909
Centro Universitário Serra dos Órgãos, Brasil
E-mail: jp.sp2008@ hotmail.com
Isabella Miranda Esteves Orsi
ORCID: https://orcid.org/0000-0002-4651-4926
Universidade José do Rosário Vellano, Brasil
E-mail: orsiisa@ hotmail.com
Alex Sandro Leão
ORCID: https://orcid.org/0000-0001-5175-0782
Universidade Federal de Alfenas, Brasil
E-mail: asleao@ uol.com.br
Talles Dias Orsi
ORCID: https://orcid.org/0000-0002-0193-5735
Universidade José do Rosário Vellano, Brasil
E-mail: tallesorsi64@ gmail.com
Daniela Maggioni Pereira Leão
ORCID: https://orcid.org/0000-0001-5240-2640
E-mail: dmaggioni@ bol.com.br
Paula Miranda Esteves Orsi
Casa de Caridade de Alfenas “Nossa Senhora Perpéto Socorro, Brasil
ORCID: https://orcid.org/0000-0003-4820-7118
Universidade José do Rosário Vellano, Brasil
E-mail: paulameorsi@hotmail.com

\section{Resumo}

O sistema intrauterino (SIU) é um método contraceptivo eficaz, reversível e muito popular em países emergentes. Entretanto, em alguns casos, podem ocorrer alguns agravantes, como o deslocamento do SIU que é uma rara complicação. A migração do SIU pode causar a perfuração uterina, tanto durante como depois da inserção, que pode ser assintomática ou apresentar sintomas clínicos que variam de sangramento anormal até formação de fístula. Relatamos o caso de uma paciente de 31 anos que possuía SIU e se queixava de dor de intensidade moderada em região hipogástrica. Durante uma histerectomia, foi constatado que o SIU estava em localização extrauterina.

Palavras-chave: Contracepção; Métodos contraceptivos; Perfuração uterina; Histerectomia; Tomografia computadorizada.

\begin{abstract}
The intrauterine system (IUS) is an effective and reversible contraceptive method, very popular in emerging countries. However, in some cases, aggravating factors may occur, such as displacement of the IUS, which is a rare complication. The migration of the IUS may cause uterine perforation, either during or after insertion, which can be asymptomatic or present clinical symptoms ranging from abnormal bleeding to fistula formation. We report a case of a 31-year-old patient, IUS user, who complained of moderate pain in the hypogastric region. During a hysterectomy, it was found that the IUS was in extrauterine location.
\end{abstract}

Keywords: Contraception; Contraceptive methods; Uterine perforation; Hysterectomy; Computed tomography.

\section{Resumen}

El sistema intrauterino (SIU) es un método anticonceptivo eficaz, reversible y muy popular en los países emergentes. Sin embargo, en algunos casos, pueden aparecer factores agravantes, como el desplazamiento del SIU, que es una complicación poco común. La migración del SIU puede ocasionar perforación uterina, ya sea durante o después de la inserción, que puede ser asintomática o presentar síntomas clínicos que van desde sangrado anormal hasta formación de fístula. Presentamos el caso de una paciente de 31 años, usuario de SIU, que consultaba por dolor moderado en la región hipogástrica. Durante una histerectomía, se encontró que el SIU estaba en una localización extrauterina.

Palabras clave: Anticoncepción; Métodos anticonceptivos; Perforación uterina; Histerectomía; Tomografía computadorizada. 


\section{Introdução}

O sistema intrauterino (SIU) é o método contraceptivo reversível mais utilizado no mundo atualmente pelas mulheres (Nouioui, et al., 2020; Teixeira et al., 2019). Seu uso apresenta-se com maior incidência nos países em desenvolvimento, sobretudo na Ásia Oriental (Sun et al., 2018). Entre as contraindicações de seu uso, estão as malformações uterinas, miomas submucosos e processos inflamatórios pélvicos (Sun et al., 2018; Teixeira et al., 2019).

Apesar de ser amplamente utilizado, a colocação do SIU pode causar complicações, sendo a perfuração uterina a mais grave delas (Ferguson, Costescu, Jamieson, \& Jong, 2016; Nouioui et al., 2020). Na maioria dos casos, a perfuração ocorre no momento da inserção, no entanto, pode haver migração subsequente e deslocamento do mesmo na cavidade. Essa complicação foi primeiramente descrita por Murphy em 1933 e, atualmente, apresenta taxa de incidência baixa que varia de 0,3 a 3,6 casos a cada 1.000 inserções, podendo ser considerada uma complicação rara (Heinemann, Reed, Moehner, \& Minh, 2015, Nouioui, et al., 2020; Yang, Duan, \& Wu, 2017).

A perfuração uterina pode ser assintomática e descoberta apenas no acompanhamento de rotina ou em conexão com uma gravidez indesejada (Marchi et al., 2012). Por outro lado, ela pode causar muita dor, sangramento anormal ou formação de fístula (Ho \& Chamsy, 2014; Marchi et al., 2012; Rowlands, Oloto, \& Horwell, 2016). O diagnóstico de perfuração uterina não pode ser excluído quando o fio do SIU não é visível durante o exame ginecológico (Marchi et al., 2012).

Este é um estudo qualitativo descritivo na forma de relato de caso. Relatos de caso são fontes de informação importantes que podem fornecer subsídios fundamentais para o melhor tratamento de pacientes em situações onde a entidade diagnosticada é rara (Goldim \& Fleck, 2010; Packer, Berger, \& Mookherjee, 2017; Yoshida, 2007).

\section{Relato de Caso}

Paciente de 31 anos, G2P2C2 e D.U.M 05/03/2020 compareceu ao ambulatório de ginecologia para consulta, referindo dor de intensidade moderada em região hipogástrica. A paciente trazia exames de ultrassonografia transvaginal que sugeria a presença de sistema intrauterino (SIU) que ocupava toda cavidade uterina e que estava normoposicionado. Porém, a paciente possuía uma ressonância magnética da pelve que referia cavidade uterina livre de SIU, existindo uma imagem linear projetada da cicatriz cirúrgica uterina (cicatriz da cesariana) para o interior do miométrio paramediano direito, com partes exteriorizadas para a região anexial, indicando suspeita de SIU intramural uterino. Foi, então, realizado exame ginecológico, sendo observado o fio do SIU. Devido à divergência de laudos e a observação do fio, foi solicitado uma tomografia computadorizada de abdômen (Alves, 2019).

O resultado da tomografia mostrava que havia a presença de SIU deslocado superior e lateralmente à direita em relação a cavidade intrauterina, com uma aparente transfixação mural de uma das hastes e outra na região cornual, com segmento paralelo à luz da tuba uterina. Com este resultado, optou-se consensualmente pela realização de uma histerectomia (Silva Filho, Triginelli, \& Traiman, 2010). Durante o procedimento, foi visto que o SIU estava localizado na região anexial direita extrauterina, confirmando os dados obtidos pela tomografia computadorizada. Após a retirada, a peça foi enviada para exame anatomopatológico, no qual, aos cortes, foi evidenciado o SIU extrauterino envolto por extensas faixas de fibrose.

É interessante observar, na Figura 1, o SIU (modelo Mirena® que libera levonorgestrel) exteriorizado e localizado na parede lateral do útero em região ovariana. 
Figura 1. Fotografias do útero perfurado pelo sistema intrauterino (SIU), realizadas após histerectomia. ( $A$ ) Parte do útero com o SIU localizado à esquerda e escala no canto inferior direito da foto; e $(B)$ acima, parte do útero com SIU localizado inferiormente e, abaixo, porção anterior do útero.
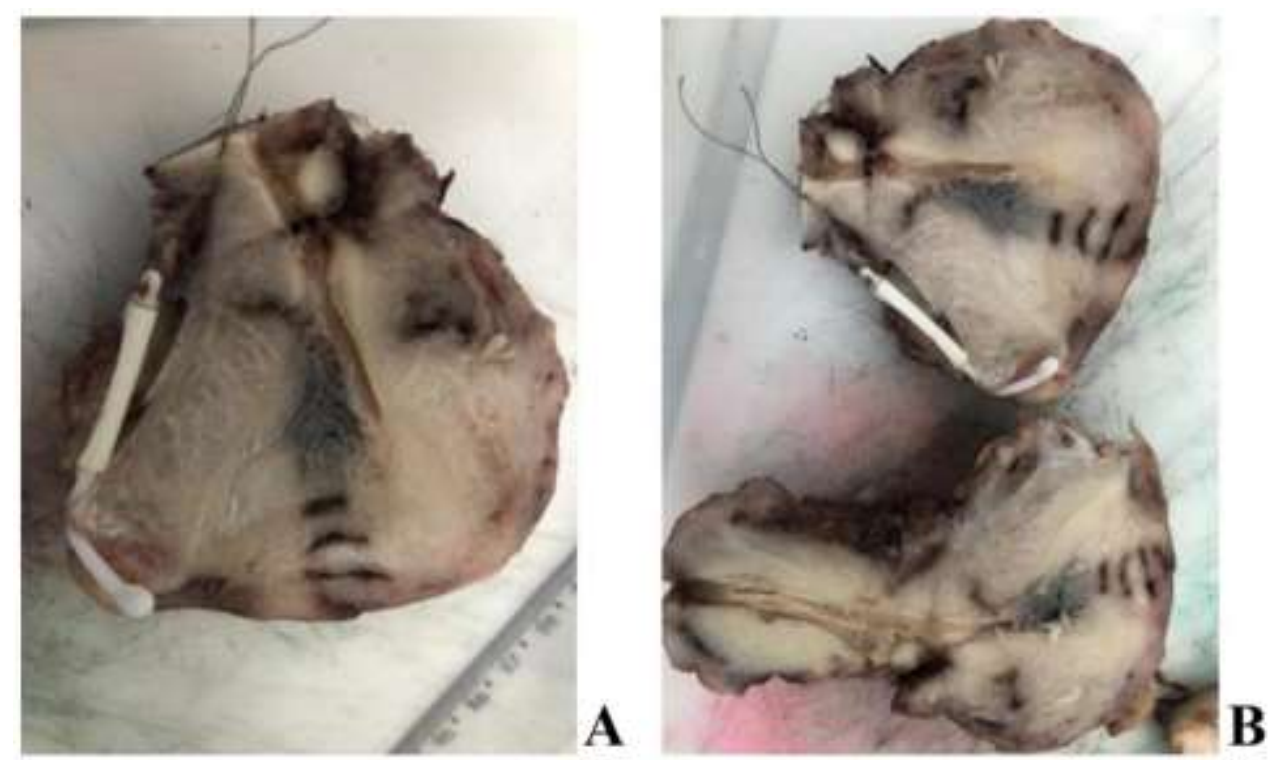

Fonte: Autores.

\section{Considerações Éticas}

O estudo foi realizado de acordo com os princípios éticos da Declaração de Helsinque (1964 e suas versões posteriores) da Associação Médica Mundial. A paciente assinou o Termo de Consentimento Livre e Esclarecido para o procedimento e uso de dados clínicos e registros fotográficos para fins científicos e publicação. O anonimato da paciente foi respeitado.

\section{Discussão}

O SIU é um método de contracepção muito eficaz, reversível, econômico e de longa duração, sendo também conhecido como DIU (dispositivo intrauterino) e mais usado em países emergentes (Kaplanoğlu, Bülbül, Yüce, Kaplanoğlu, \& Aban, 2015). A perfuração uterina por SIU é uma complicação grave e rara que pode ocorrer durante sua inserção ou posteriormente. Pode haver migração ou deslocamento geralmente para as cavidades abdominal e pélvica, podendo afetar o funcionamento de vários órgãos vizinhos, como a bexiga, o intestino delgado, o retossigmóide e o apêndice vermiforme (Coelho, Gonçalves, \& Graf, 2003; Rowlands et al., 2016).

Entre os dois tipos de dispositivos disponíveis, aqueles que liberam levonorgestrel e os de cobre, o primeiro está associado à perfuração uterina em 0,3 a 3,6 casos a cada 1000 inserções e o segundo, 1,1 caso a cada 1000 inserções (HarrisonWoolrych et al., 2003; Heinemann et al., 2015, Nouioui et al., 2020; Yang et al., 2017). A incidência de perfuração é maior nos primeiros 6 meses após a colocação (Sun et al., 2018). As causas dessas perfurações ainda não são totalmente conhecidas, sendo as mais prováveis o período pós-parto imediato e a amamentação, visto que o útero mantém sua contração reativa repetitiva devido à ocitocina com associação a alta do estrogênio (Coelho et al., 2003; Kaislasuo, Suhonen, Gissler, Lähteenmäki, \& Heikinheimo, 2012; Kaplanoğlu et al., 2015; Rowlands et al., 2016; Teixeira et al., 2019; Yang et al., 2017).

Um dos maiores problemas da perfuração e da migração do SIU é que, em muitos casos, eles são assintomáticos, sendo uma média de 30\% descobertos apenas na gravidez (Barnett, Moehner, Minh, \& Heinemann, 2017; Nouioui et al., 2020; Rowlands et al., 2016; Yang et al., 2017). Os sintomas dependem da localização do SIU, e podem gerar desde hematúria e 
cálculos renais até dor, sangramentos abdominais e fístulas. (Ho \& Chamsy, 2014; Rowlands et al., 2016)

O diagnóstico é feito por exame ginecológico, ultrassonografia, radiografia abdominal e, caso necessário por recomendação médica, tomografia computadorizada, visto que fornece informações precisas sobre a localização e sobre os danos do SIU (Agacayak et al., 2015; Alves, 2017; Coelho et al., 2003; Ho \& Chamsy, 2014; Rowlands et al., 2016; Teixeira et al., 2019).

\section{Considerações Finais}

No caso relatado, foi usado como tratamento a histerectomia e não foi necessário realizar a laparoscopia que é considerada a primeira linha de tratamento, pois o SIU não migrou para outros órgãos além da região uterina (Agacayak et al., 2015; Coelho et al., 2003; Stovall, 2011; Yang et al., 2017). Estudos futuros baseados em conjuntos de dados retrospectivos seriam interessantes, pois poderiam revelar as causas principais e complicações mais frequentes decorrentes da perfuração uterina por SIU na população brasileira.

\section{Referências}

Agacayak, E., Tunc, S. Y., Icen, M. S., Oguz, A., Ozler, A., Turgut, A., \& Basaranoglu, S. (2015). Evaluation of predisposing factors, diagnostic and treatment methods in patients with translocation of intrauterine devices. Journal of Obstetrics and Gynaecology Research, 41(5), 735-741.

Alves, R. D. (2019). Influência do posicionamento do sistema intrauterino liberador de levonorgestrel no padrão de sangramento uterino (Dissertação de Mestrado). Salvador: Escola Bahiana de Medicina e Saúde Pública.

Barnett, C., Moehner, S., Minh, T. D., \& Heinemann, K. (2017). Perforation risk and intra-uterine devices: results of the EURAS-IUD 5-year extension study. The European Journal of Contraception \& Reproductive Health Care, 22(4), 424-428.

Coelho, J. C., Gonçalves, C. G., \& Graf, C. M. (2003). Laparoscopic treatment of peri-appendicitis caused by intrauterine contraceptive device. Arquivos de Gastroenterologia, 40(1), 45-46.

Ferguson, C. A., Costescu, D., Jamieson, M. A., \& Jong, L. (2016). Transmural migration and perforation of a levonorgestrel intrauterine system: a case report and review of the literature. Contraception, 93(1), 81-86.

Goldim, J. R., \& Fleck, M. P. (2010). Ética e publicação de relatos de caso individuais. Brazilian Journal of Psychiatry, 32(1), 2-3.

Harrison-Woolrych, M., Ashton, J., \& Coulter, D. (2003). Uterine perforation on intrauterine device insertion: Is the incidence higher than previously reported? Contraception, 67(1), 53-56.

Heinemann, K., Reed, S., Moehner, S., \& Minh, T. D. (2015). Risk of uterine perforation with levonorgestrel-releasing and copper intrauterine devices in the European Active Surveillance Study on Intrauterine Devices. Contraception, 91(4), 274-279.

Ho, K. A., \& Chamsy, D. J. (2014). Perforated intraperitoneal intrauterine contraceptive devices: Diagnosis, management and clinical outcomes. Journal of Minimally Invasive Gynecology, 21(4), 596-601.

Kaislasuo, J., Suhonen, S., Gissler, M., Lähteenmäki, P., \& Heikinheimo, O. (2012). Intrauterine contraception: incidence and factors associated with uterine perforation: A population-based study. Human Reproduction, 27(9), 2658-2663.

Kaplanoğlu, M., Bülbül, M., Yüce, T., Kaplanoğlu, D., \& Aban, M. (2015). Mislocated extrauterine intrauterine devices: diagnosis and surgical management. Journal of The Turkish-German Gynecological Association, 16(2), 91-95.

Marchi, N. M., Castro, S., Hidalgo, M. M., Hidalgo, C., Monteiro-Dantas, C., Villarroeal, M., \& Bahamondes L. (2012). Management of missing strings in users of intrauterine contraceptives. Contraception, 86(4):354-8.

Nouioui, M. A, Taktak, T., Mokadem, S., Mediouni, H., Khiari, R., \& Samir Ghozzi, S. (2020). A mislocated intrauterine device migrating to the urinary bladder: An uncommon complication leading to stone formation. Case Report in Urology, 2020, 1-4.

Packer, C., Berger, G., \& Mookherjee, S. (2017). Writing case reports: A practical guide from conception to publication. Switzerland: Springer International Publishing.

Rowlands, S., Oloto, E., \& Horwell, D. H. (2016). Intrauterine devices and risk of uterine perforation: Current perspectives. Open Access Journal of Contraception, 7, 19-32.

Silva Filho, A. L., Triginelli, S.A., \& Traiman, P. (2010). Manual de Cirurgia Ginecológica. MedBook.

Stovall, D. W. (2011). Alternatives to hysterectomy: focus on global endometrial ablation, uterine fibroid embolization, and magnetic resonance-guided focused ultrasound. Menopause, 18(4), 437-444.

Sun, X., Xue. M., Deng, X, Lin, Y., Tan, Y., \& Wei, X. (2018). Clinical characteristic and intraoperative findings uterine perforation patients in using of 
Research, Society and Development, v. 10, n. 5, e26410514585, 2021

(CC BY 4.0) | ISSN 2525-3409 | DOI: http://dx.doi.org/10.33448/rsd-v10i5.14585

intrauterine devices (IUDs). Gynecological Surgery, 15(1), 3.

Teixeira, J. P., Rosa Neto, F. R., Costa, R. E., Cintra, T. R, Costa, C. L., Vieira, I. L., \& Gomes, D. A. (2019). Paciente com perfuração uterina assintomática após inserção de dispositivo intrauterino: Relato de caso e revisão de literatura. Revista de Medicina e Saúde de Brasília, 8(1), 18-24.

Yang X., Duan X., \& Wu T. (2017). Ureteric obstruction caused by a migrated intrauterine device. Urology Case Reports, 10, 33-35.

Yoshida, W. B. (2007). Redação do relato de caso. Jornal Vascular Brasileiro, 2007;6(2):112-113. 